\title{
TRANSPORT
}

\section{МАТМОДЕЛЬ ГАЗОВОГО ДВИГАТЕЛЯ, ИСПОЛЬЗУЕМОГО В КАЧЕСТВЕ ПРИВОДА МОБИЛЬНОГО ТРАНСПОРТНОГО СРЕДСТВА}

\author{
Манойло В. М., кандидат технических наук, доцент \\ Украина, Харьков, Харьковский нацииональньій \\ технический университет сельского \\ хозяйства имени Петра Василенко
}

DOI: https://doi.org/ 10.31435/rsglobal_ws/30092018/6130

\begin{abstract}
ARTICLE INFO
Received: 15 July 2018

Accepted: 26 September 2018

Published: 30 September 2018

\section{KEYWORDS}

control object,

internal combustion engine,

differential,

dimensionless and operator equations.

ABSTRACT

The linear differential equation of the internal combustion engine (ICE) itself, by means of which it is possible to investigate the dynamic characteristics (transients) and throttle response of the new generation of gas engines, used as a drive for mobile vehicles, is given. At present, the need has arisen to create a new generation of ICS control systems that would combine the principles of robust and adaptive management and provide a specified quality of management in the conditions of structural or parametric uncertainty of the control facility itself, uncertainty of external conditions and management goal uncertainty. The most important contour in the engine control system is the crankshaft speed regulator. It has a determining effect on the technical, economic and environmental performance of ICE. The resulting differential equation describes the transient processes of the angular velocity variation of the crankshaft of the gas engine of vehicles in a wide range of speed and load modes of operation.
\end{abstract}

Citation: Манойло В. М. (2018) Matmodel Gazovogo Dvigatelya Ispolzuemogo v Kachestve Privoda Mobilnogo Transportnogo Sredstva. World Science. 9(37). doi: 10.31435/rsglobal_ws/30092018/6130

Copyright: (C) 2018 Манойло В. М. This is an open-access article distributed under the terms of the Creative Commons Attribution License (CC BY). The use, distribution or reproduction in other forums is permitted, provided the original author(s) or licensor are credited and that the original publication in this journal is cited, in accordance with accepted academic practice. No use, distribution or reproduction is permitted which does not comply with these terms.

Введение. В качестве объекта регулирования автором предложена математическая модель изменения угловой скорости вращения коленчатого вала газового двигателя с искровым зажиганием, позволяющая расчетно-экспериментальным методом исследовать динамические и технико-экономические характеристики транспортного ДВС.

Цель работы. В литературе широко представлены решения задач расчета переходных процессов, без надувных и надувных вариантов двигателей, с использованием дифференциального уравнения (ДУ) изменения угловой скорости вращения вала ДВС [10, 31 и др.]. Все они относятся к дизелям, оборудованным гидромеханической топливной аппаратурой (ТА), либо аккумуляторной электромеханической ТА.

ДУ, описывающее приращение угловой скорости вращения коленчатого вала газового ДВС для привода конвертированного АТС в литературных источниках не обнаружено. Устранение этого информационного пробела является целью данной статьи.

Анализ известных матмоделей. При создании систем управления ДВС основное внимание традиционно уделяется установившимся режимам работы. Это обусловлено тем, что 
для оптимизации параметров систем управления используют снятые экспериментально статические характеристики.

Между тем, в процессе интенсивного городского движения, карьерных, дорожностроительных условиях работы неустановившиеся режимы работы ДВС составляют 80-97\% . Работа ДВС при неустановившихся режимах приводит к уменьшению моторесурса до 50\%, увеличению расхода топлива более чем на 40\% [1]. Еще большее значение оптимизация работы ДВС приобретает при ужесточении требований к экологическим характеристикам транспортных средств.

В технической литературе имеются работы [1, 6 и 7], где объектом исследования является собственно дизель. Поэтому, данные работы не корректно использовать для исследования, например, газовых двигателей. У ДВС с искровым зажиганием совершенно иной рабочий процесс сгорания по сравнению с дизельными двигателями.

Таким образом, в настоящее время созрела необходимость в создании нового поколения систем управления ДВС, которые сочетали бы в себе принципы робастного и адаптивного управления и обеспечивали бы заданное качество управления в условиях структурной либо параметрической неопределенности самого объекта управления, неопределенности внешних условий и неопределенности цели управления.

Наиболее ответственным контуром в системе управления ДВС является регулятор частоты вращения коленчатого вала. Он оказывает определяющее влияние на техникоэкономические и экологические показатели работы ДВС.

\section{Динамические свойства ДВС}

В установившемся режиме работы крутящий момент ДВС $M$ равен моменту сопротивления $M_{C}$ В этих условиях установившаяся угловая скорость вращения коленчатого вала (КВ) определяется точкой пересечения скоростной характеристики ДВС $M=f(\omega)$ и характеристики потребителя $M_{C}=f(\omega)$.

В том случае, если крутящий момент и/или момент сопротивления получают приращения, появляется избыток либо недостаток крутящего момента, приводящий к изменению угловой скорости вращения КВ

Для пояснення фізичної сутності впливу вібрації на поведінку грунтів під навантаженням висунутий цілий ряд гіпотез. Найбільш широкого поширення набуло представлення грунтів у вигляді коливальної системи, яка, в загальному випадку, має в'язкий опір коливань. При цьому опір коливань приймається пропорційним швидкості коливання.

У цьому випадку переміщення тіла, що здійснює плоскі горизонтально-обертальні коливання, описується системою диференціальних рівнянь виду:

$$
J \frac{d \omega}{d t}=(M+\Delta M)-\left(M_{C}+\Delta M_{C}\right)
$$

поскольку $M=M_{C}$, тогда выражение (1) можно упростить.

$$
J \frac{d \omega}{d t}=\Delta M-\Delta M_{C}
$$

где $J$ - суммарный момент инерции, приведенный к оси вращения КВ, характеризует способность ДВС аккумулировать в себе механическую энергию, кг $\mathbf{M}^{2}$;

$\Delta M$ - приращение крутящего момента ДВС, Нм;

$\Delta M_{C}$ - соответственно приращение момента сопротивления ДВС, Нм.

Эффективный удельный расход топлива ДВС (кг/кВт·ч), определяется с помощью следующей зависимости

$$
g_{e}=\frac{G_{u a c}}{N_{e}}=\frac{3600}{H_{u} \cdot \eta_{i} \cdot \eta_{m}}
$$

где $G_{\text {час }}$ - часовой расход топлива ДВС, кг/ч;

$N_{e}$ - эффективная мощность ДВС, кВт; 
$H_{u}$ - теплота сгорания топлива, МДж/кг;

$\eta_{i}$ - индикаторный КПД ДВС;

$\eta_{m}$ - механический КПД, учитывающий все внутренние потери двигателя.

Следовательно, преобразовав выражение (3) можно записать выражение для эффективной мощности двигателя (кВт):

$$
N_{e}=\frac{H_{u} \cdot i_{\text {Д }}}{\pi \cdot \tau_{\text {Д }}} \cdot \eta_{i} \cdot \eta_{m} \cdot \omega \cdot g_{u}
$$

где $i_{\text {д }}$ - число цилиндров двигателя;

$\omega$ - угловая скорость вращения коленчатого вала, рад/с;

$\tau_{\text {д }}-$ тактность двигателя;

$g_{u}$ - цикловая подачи топлива ДВС, кг/цикл.

Цикловую подачу топлива (кг/цикл): если двигатель, оборудованный стандартной газотопливной аппаратурой, то необходимую зависимость можно записать соотношением

$$
q_{u}=\frac{q_{e} \cdot N_{e} \cdot \pi \cdot \tau_{\text {д }}}{3600 \cdot i_{\text {д }} \cdot \omega}
$$

а, для ДВС, оборудованного топливной аппаратурой (ТА) с распределенным дозированием топлива во впускной коллектор, статическую характеристику запишем следующим выражением

$$
q_{u}=f \cdot \rho_{t} \cdot \vartheta \cdot \tau_{b}
$$

где: $f$ - площадь проходного сечения жиклера ТА, мм²;

$\rho_{t}-$ плотность топлива, кг $/ \mathrm{m}^{3}$;

$\tau_{b}$ - время подачи дозатором топлива (газа) в ДВС, с;

$\vartheta$ - скорость движения топлива в жиклере дозатора, м/с.

Часовой расход топлива (кг/ч), определяется следующим выражением

$$
G_{\text {час }}=\frac{3600 \cdot i_{\text {Д }}}{\pi \cdot \tau_{\text {д }}} \cdot \omega \cdot g_{u}
$$

Учитывая, что крутящий момент двигателя $(\mathrm{H} \cdot \mathrm{m})$ записывается в виде

$$
M=10^{3} \cdot \frac{N_{e}}{\omega}
$$

подставим в него выражение (4) тогда получим,

$$
M=\frac{10^{3} \cdot H_{u} \cdot i_{\text {Д }}}{\pi \cdot \tau_{\text {Д }}} \cdot \eta_{i} \cdot \eta_{m} \cdot g_{u}=K \cdot \eta_{i} \cdot \eta_{m} \cdot g_{u}
$$

топлива.

где $K=\frac{10^{3} \cdot H_{u} \cdot i_{\text {Д }}}{\pi \cdot \tau_{\text {д }}}-$ коэффициент, зависящий от конструкции двигателя и вида

Параметры $\eta_{i}, \eta_{i i}, \eta_{m}$ и $g_{u}$, входящие в последние выражения, зависят от режима работы ДВС.

Приведенные выше выражения определяют значения крутящего момента двигателя на установившихся режимах работы. Параметры работы двигателей на неустановившихся режимах заметно отличаются (на 10-15\%) от соответствующих им параметров на сходственных установившихся режимах. Сходственными называют режимы, при которых положение органов управления и средняя за цикл угловая скорость КВ равны их мгновенным значениям соответствующего неустановившегося режима работы. 
Таким образом, уравнения, характеризующие работу ДВС в установившихся режимах, могут использоваться только для изучения характера влияния различных параметров на работу двигателей и для предварительной оценки величины этого влияния. Построенная, на их основе, математическая модель ДВС в дальнейшем подлежит параметрической идентификации.

Поскольку зависимость $M=f\left(g_{u}, \eta_{i}, \eta_{m}\right)$ является гладкой непрерывной функцией, дифференцируемой во всей области допустимых значений своих параметров, рассмотрим ее разложение в ряд Тейлора в окрестности величины крутящего момента, соответствующего установившемуся режиму

$$
\begin{aligned}
M\left(g_{u}, \eta_{i}, \eta_{m}\right)= & M\left(g_{u 0}, \eta_{i 0}, \eta_{m 0}\right)+\frac{\partial M\left(g_{u 0}, \eta_{i 0}, \eta_{m 0}\right)}{\partial g_{u}} \cdot\left(g_{u}-g_{u 0}\right)+\frac{\partial^{2} M\left(g_{u 0}, \eta_{i 0}, \eta_{m 0}\right)}{\partial g_{u}{ }^{2}} \cdot \frac{\left(g_{u}-g_{u 0}\right)^{2}}{2 !}+\ldots \\
& +\frac{\partial M\left(g_{u 0}, \eta_{i 0}, \eta_{m 0}\right)}{\partial \eta_{i}} \cdot\left(\eta_{i}-\eta_{i 0}\right)+\frac{\partial^{2} M\left(g_{u 0}, \eta_{i 0}, \eta_{m 0}\right)}{\partial \eta_{i}{ }^{2}} \cdot \frac{\left(\eta_{i}-\eta_{i 0}\right)^{2}}{2 !}+\ldots \\
& +\frac{\partial M\left(g_{u 0}, \eta_{i 0}, \eta_{m 0}\right)}{\partial \eta_{m}} \cdot\left(\eta_{m}-\eta_{m 0}\right)+\frac{\partial^{2} M\left(g_{u 0}, \eta_{i 0}, \eta_{m 0}\right)}{\partial \eta_{m}{ }^{2}} \cdot \frac{\left(\eta_{m}-\eta_{m 0}\right)^{2}}{2 !}+\ldots,
\end{aligned}
$$

где $g_{u 0}, \eta_{i 0}$ и $\eta_{m 0}$ - соответственно цикловая подача топлива, индикаторный и механический КПД, соответствующие установившимся режимам работы ДВС.

При малых значениях отклонений режимов работы ДВС от установившегося можно ограничиться лишь первыми членами разложения. В этом случае приращение крутящего момента представляет собой линейную функцию приращений своих аргументов

$$
\begin{aligned}
\Delta M & =M\left(g_{u}, \eta_{i}, \eta_{m}\right)-M\left(g_{u 0}, \eta_{i 0}, \eta_{m 0}\right)= \\
& =\frac{\partial M\left(g_{u 0}, \eta_{i 0}, \eta_{m 0}\right)}{\partial g_{u}} \cdot \Delta g_{u}+\frac{\partial M\left(g_{u 0}, \eta_{i 0}, \eta_{m 0}\right)}{\partial \eta_{i}} \cdot \Delta \eta_{i}+\frac{\partial M\left(g_{u 0}, \eta_{i 0}, \eta_{m 0}\right)}{\partial \eta_{m}} \cdot \Delta \eta_{m},
\end{aligned}
$$

где $\Delta g_{u}=g_{u}-g_{u 0}-$ приращение цикловой подачи топлива ДВС, кг/цикл; $\Delta \eta_{i}=\eta_{i}-\eta_{i 0}-$ приращение индикаторного КПД двигателя; $\Delta \eta_{m}=\eta_{m}-\eta_{m 0}-$ приращение механического КПД ДВС.

Частные производные, входящие в полученные выражения, можно определить дифференцированием выражений для крутящего момента в точке, соответствующей установившемуся режиму работы:

$$
\begin{aligned}
& \frac{\partial M\left(g_{u 0}, \eta_{i 0}, \eta_{m 0}\right)}{\partial g_{u}}=K \cdot \eta_{i 0} \cdot \eta_{m 0}=\frac{M_{0}}{g_{u 0}} \\
& \frac{\partial M\left(g_{u 0}, \eta_{i 0}, \eta_{m 0}\right)}{\partial \eta_{i}}=K \cdot g_{u 0} \cdot \eta_{m 0}=\frac{M_{0}}{\eta_{i 0}} \\
& \frac{\partial M\left(g_{u 0}, \eta_{i 0}, \eta_{m 0}\right)}{\partial \eta_{m}}=K \cdot g_{u 0} \cdot \eta_{i 0}=\frac{M_{0}}{\eta_{m 0}}
\end{aligned}
$$

где $M_{0}$ - крутящий момент двигателя на установившемся режиме, $\mathrm{H} \cdot \mathrm{M}$.

В результате выражение для приращения крутящего момента примет вид

$$
\Delta M=K \cdot \eta_{i 0} \cdot \eta_{m 0} \cdot \Delta g_{u}+K \cdot g_{u 0} \cdot \eta_{m 0} \cdot \Delta \eta_{i}+K \cdot g_{u 0} \cdot \eta_{i 0} \cdot \Delta \eta_{m}
$$

Основными факторами, определяющими значение индикаторного КПД двигателя, являются: коэффициент избытка воздуха $\alpha$, разрежение рабочего тела во впускном коллекторе 
двигателя $\Delta p_{a}$ и коэффициент наполнения цилиндров $\eta_{v}$ ДВС. Отклонение данных параметров от их значений в установившемся режиме вызывает приращение индикаторного КПД

$$
\Delta \eta_{i}=\frac{\partial \eta_{i}\left(\alpha_{0}, p_{a 0}, \eta_{v 0}\right)}{\partial \alpha} \cdot \Delta \alpha+\frac{\partial \eta_{i}\left(\alpha_{0}, p_{a 0}, \eta_{v 0}\right)}{\partial\left(\Delta p_{a}\right)} \cdot \Delta\left(\Delta p_{a}\right)+\frac{\partial \eta_{i}\left(\alpha_{0}, p_{a 0}, \eta_{v 0}\right.}{\partial \eta_{v}} \cdot \Delta \eta_{v}
$$

где $\Delta \alpha=\alpha-\alpha_{0}$ - отклонение коэффициента избытка воздуха от его значения в установившемся режиме работы ДВС;

$\Delta\left(\Delta p_{a}\right)=\Delta p_{a}-\Delta p_{a 0}-$ приращение разрежения во впускном коллекторе относительно его значения в установившемся режиме работы двигателя, МПа;

$\Delta \eta_{v}=\eta_{v}-\eta_{v 0}-$ отклонение коэффициента наполнения цилиндра от его значения в установившемся режиме работы ДВС;

$\alpha_{0}, \eta_{v 0}-$ соответственно коэффициент избытка воздуха и коэффициент наполнения цилиндра при установившемся режиме работы ДВС;

$p_{a 0}-$ разряжение воздуха во впускном коллекторе двигателя в установившемся режиме работы ДВС, МПа.

Учитывая взаимосвязь неустановившихся и сходственных установившихся режимов работы, частные производные, входящие в последнее уравнение, могут быть определены дифференцированием выражения для индикаторного КПД:

$$
\eta_{i}=8,314 \frac{L_{0} \cdot \alpha \cdot p_{i} \cdot T_{a}}{H_{u} \cdot \eta_{v} \cdot p_{a}}
$$

где $L_{0}$ - количество воздуха, теоретически необходимое для сгорания 1 кг топлива, кмоль;

$p_{i}$ - среднее индикаторное давление, МПа;

$T_{a}$ - температура воздуха во впускном коллекторе двигателя, К. Будем считать

$T_{a}=$ const; тогда $p_{a}$ - разряжение воздуха во впускном коллекторе, МПа

$$
p_{a}=p_{0}-\Delta p_{a 0}-\Delta\left(\Delta p_{a}\right)
$$

где $p_{0}$ - давление окружающей среды, МПа.

При этом производные должны определяться в точке, соответствующей выбранному равновесному режиму

$$
\begin{gathered}
\frac{\partial \eta_{i}\left(\alpha_{0}, p_{a 0}, \eta_{v 0}\right)}{\partial \alpha}=8,314 \cdot \frac{L_{0} \cdot p_{i} \cdot T_{a}}{H_{u} \cdot \eta_{v 0} \cdot p_{a 0}} \\
\frac{\partial \eta_{i}\left(\alpha_{0}, p_{\grave{a} 0}, \eta_{v 0}\right)}{\partial\left(\Delta p_{\grave{a}}\right)}=8,314 \cdot \frac{L_{0} \cdot \alpha_{0} \cdot p_{\grave{i}} \cdot T_{\grave{a}}}{H_{u} \cdot \eta_{v 0} \cdot p_{\grave{a} 0}^{2}} \\
\frac{\partial \eta_{i}\left(\alpha_{0}, p_{\grave{a} 0}, \eta_{v 0}\right)}{\partial \eta_{v}}=-8,314 \cdot \frac{L_{0} \cdot \alpha_{0} \cdot p_{i} \cdot T_{\grave{a}}}{H_{u} \cdot \eta_{v 0}^{2} \cdot p_{\grave{a} 0}}
\end{gathered}
$$

Коэффициент избытка воздуха ДВС выразим следующей зависимостью

$$
\alpha=\frac{G_{u B}}{L_{0} \cdot g_{u}}
$$

где $G_{u B}$ - цикловая подача воздуха в ДВС, кг/цикл

$$
G_{u B}=V_{h} \cdot \rho_{a} \cdot \eta_{v}
$$

$V_{h}$ - рабочий объем цилиндров ДВС, л; 
$\rho_{a}$ - плотность воздуха во впускном коллекторе, которую будем считать равной плотности воздуха в цилиндрах ДВС, кг/ $\mathrm{M}^{3}$.

При небольших значениях разрежения в цилиндрах ДВС рабочего тела во впускных коллекторах, используется уравнение Менделеева - Клапейрона (для идеального газа):

$$
\rho_{a}=\frac{\mu}{R \cdot T_{a}} \cdot p_{a}
$$

где $\mu=28,8$ - молекулярная масса воздуха, кг/моль;

$R$ - характеристическая газовая постоянная воздуха, кДж/(кг·К).

Следовательно, коэффициент избытка воздуха в цилиндрах ДВС, можно выразить следующей зависимостью, используя зависимости (22 и 23)

$$
\alpha=\frac{V_{h} \cdot \eta_{v} \cdot \mu}{L_{0} \cdot q_{u} \cdot R \cdot T_{a}} \cdot p_{a}
$$

В полученных формулах, параметры $V_{h}$ и $L_{0}$, являются постоянными для всех режимов работы двигателя, $T_{a}$ изменяется достаточно медленно и ее влиянием на динамику ДВС можно пренебречь. Поэтому будем считать для ДВС

$$
\alpha=f\left(\eta_{v}, q_{u}, p_{a}\right)
$$

После разложения полученной зависимости в ряд Тейлора в окрестности установившегося режима, учитывая, что $p_{a}=p_{0}-\Delta p_{a}$. Получим линеаризованное выражение для приращения коэффициента избытка воздуха

$$
\Delta \alpha=\frac{\partial \alpha\left(\eta_{v 0}, q_{0}, p_{a 0}\right)}{\partial \eta_{v}} \cdot \Delta \eta_{v}+\frac{\partial \alpha\left(\eta_{v 0}, q_{0}, p_{a 0}\right)}{\partial q} \cdot \Delta q+\frac{\partial \alpha\left(\eta_{v 0}, q_{0}, p_{a 0}\right)}{\partial\left(\Delta p_{a}\right)} \cdot \Delta\left(\Delta p_{a}\right)
$$

Частные производные должны определяться в точке, соответствующей выбранному равновесному режиму

$$
\begin{aligned}
& \frac{\partial \alpha\left(\eta_{v 0}, q_{u 0}, p_{a 0}\right)}{\partial \eta_{v}}=\frac{V_{h} \cdot \mu \cdot p_{a 0}}{L_{0} \cdot q_{u 0} \cdot R \cdot T_{a}} \\
& \frac{\partial \alpha\left(\eta_{v 0}, q_{u 0}, p_{a 0}\right)}{\partial q_{u}}=-\frac{V_{h} \cdot \eta_{v 0} \cdot \mu \cdot p_{a 0}}{L_{0} \cdot q_{u 0}^{2} \cdot R \cdot T_{a}} \\
& \frac{\partial \alpha\left(\eta_{v 0}, q_{u 0}, p_{a 0}\right)}{\partial\left(\Delta p_{a}\right)}=-\frac{V_{h} \cdot \eta_{v 0} \cdot \mu}{L_{0} \cdot q_{u 0} \cdot R \cdot T_{a}}
\end{aligned}
$$

Коэффициент наполнения цилиндров двигателя выразим следующим соотношением

$$
\eta_{v}=\frac{\varepsilon \cdot p_{a}}{(\varepsilon-1) \cdot p_{0}} \cdot \frac{T_{0}}{T_{a}\left(1+\gamma_{r}\right)}
$$

где $\varepsilon$ - степень сжатия;

$p_{a}$ - разряжение рабочего тела в цилиндре двигателя в конце впуска, МПа;

$T_{a}$ - температура свежего заряда с учетом подогрева от горячих деталей двигателя, К;

$\gamma_{r}-$ коэффициент остаточных газов.

В выражении $\eta_{v}$ величины $T_{a}$ и $p_{r}$ изменяются медленно и слабо влияют на приращение $\Delta \eta_{v}$. Величины $\varepsilon, T_{0}, p_{0}$ не зависят от режима работы двигателя.

Поскольку 


$$
p_{a}=p_{0}-\Delta p_{a}
$$

где $\Delta p_{a}$ - потери давления в цилиндре при наполнении, зависящие от режима работы ДВС. Тогда выражение для коэффициента наполнения цилиндров ДВС можно записать в виде приведения.

$$
\eta_{v}=\frac{\varepsilon \cdot p_{0}}{(\varepsilon-1) \cdot p_{0}} \cdot \frac{T_{0}}{T_{a}\left(1+\gamma_{r}\right)}-\frac{\xi}{(\varepsilon-1) \cdot p_{0}} \cdot \frac{T_{0}}{T_{a}\left(1+\gamma_{r}\right)} \cdot \Delta p_{a}=K_{h 1}-K_{h 2} \cdot \Delta p_{a}
$$

где $K_{h 1}=\frac{\varepsilon \cdot p_{0}}{(\varepsilon-1) \cdot p_{0}} \cdot \frac{T_{0}}{T_{a}\left(1+\gamma_{r}\right)} ; K_{h 2}=\frac{\xi}{(\varepsilon-1) \cdot p_{0}} \cdot \frac{T_{0}}{T_{a}\left(1+\gamma_{r}\right)}-\quad$ коэффициенты

Приращение разрежения $\Delta\left(\Delta p_{\partial}\right)$ в цилиндре ДВС в процессе наполнения при изменении скоростного режима двигателя изменяется аналогично приращению потери давления во впускном коллекторе, т. е. $\Delta\left(\Delta p_{\partial}\right) \approx \Delta\left(\Delta p_{a}\right)$. Следовательно, выражение для приращения коэффициента наполнения цилиндра, вызванного приращением разряжения во впускном коллекторе, можно записать в виде

$$
\Delta \eta_{v}=\frac{\partial \eta_{v}\left(p_{a 0}\right)}{\partial\left(\Delta p_{a}\right)} \cdot \Delta\left(\Delta p_{a}\right)=-K_{h 2} \cdot \Delta\left(\Delta p_{a}\right)
$$

Подставив полученные выражения $\Delta \alpha$ и $\Delta \eta_{v}$ в выражение для приращения индикаторного КПД $\Delta \eta_{i}$ ДВС, получим

$$
\begin{aligned}
& \Delta \eta_{i}=\frac{8,314 \cdot p_{i}}{H_{u} \cdot \eta_{v 0} \cdot p_{k 0}} \cdot\left(L_{0} \cdot \alpha_{0} \cdot T_{a} \cdot\left(\frac{1}{p_{a 0}}+\frac{K_{h 2}}{\eta_{v 0}}\right)-\frac{V_{h} \cdot \mu \cdot\left(\eta_{v 0}+K_{h 2} \cdot p_{a 0}\right)}{q_{0 u} \cdot R}\right) \cdot \Delta\left(\Delta p_{a}\right)- \\
& -\frac{8,314 \cdot p_{i} \cdot V_{h} \cdot \mu}{H_{u} \cdot q_{0 u}^{2} \cdot R} \cdot \Delta q_{u}
\end{aligned}
$$

Механический КПД двигателя может быть определен из выражения

$$
\eta_{m}=1-\frac{p_{m p}}{p_{i}}
$$

где $p_{m p}$ - среднее давление сил сопротивления (механических потерь), включающее силы трения и потери на выпуск и наполнение (насосные потери) ДВС.

По мере увеличения частоты вращения КВ числовое значение механического КПД в ДВС уменьшается за счет увеличения $p_{m p}$, вызванные увеличением инерционных сил в кривошипно-шатунном механизме и увеличением насосных потерь при смене рабочего тела, а также за счет уменьшения $p_{i}$, вызванных уменьшением коэффициента наполнения. Насосные потери при смене рабочего тела и коэффициент наполнения цилиндров зависят от величины аэродинамического сопротивления потоку воздуха, которое, в свою очередь, определяется угловым положением дроссельной заслонки.

Таким образом, можно записать

$$
\Delta \eta_{m}=\frac{\partial \eta_{m}\left(\beta_{0}, \omega_{0}\right)}{\partial \beta} \cdot \Delta \beta+\frac{\partial \eta_{m}\left(\beta_{0}, \omega_{0}\right)}{\partial \omega} \cdot \Delta \omega
$$

где $\beta_{0}$ - угловое положение дроссельной заслонки в установившемся режиме, град. пов.;

$\omega_{0}$ - угловая скорость вращения КВ ДВС в установившемся режиме, рад/с;

$\Delta \beta$ - малое отклонение углового положения дроссельной заслонки от положения установившегося режима, град. пов.; 
$\Delta \omega$ - отклонение скорости вращения КВ от значения установившегося режима, $\mathrm{paд/c.}$

Подставив выражение $\Delta \eta_{i}$ в соотношение для приращения крутящего момента $\Delta M$ для ДВС, тогда получим

$$
\begin{aligned}
& \Delta M=K \cdot q_{u 0} \cdot \eta_{i 0} \cdot \frac{\partial \eta_{m}\left(\beta_{0}, \omega_{0}\right)}{\partial \omega} \cdot \Delta \omega+ \\
& +K \cdot \eta_{m 0} \cdot\left(\eta_{i 0}-\frac{8,314 \cdot p_{i} \cdot V_{h} \cdot \mu}{H_{u} \cdot q_{u 0} \cdot R}\right) \cdot \Delta q_{u}+K \cdot q_{u 0} \cdot \eta_{i 0} \cdot \frac{\partial \eta_{m}\left(\beta_{0}, \omega_{0}\right)}{\partial \beta} \cdot \Delta \beta+ \\
& +K \cdot q_{u 0} \cdot \eta_{m 0} \cdot \frac{8,314 \cdot p_{i}}{H_{u} \cdot \eta_{v 0} \cdot p_{a 0}} \cdot\left(L_{0} \cdot \alpha_{0} \cdot T_{a} \cdot\left(\frac{1}{p_{a 0}}+\frac{K_{h 2}}{\eta_{v 0}}\right)-\frac{V_{h} \cdot \mu \cdot\left(\eta_{v 0}+K_{h 2} \cdot p_{a 0}\right)}{q_{u 0} \cdot R}\right) \cdot \Delta\left(\Delta p_{a}\right)
\end{aligned}
$$

Если, нагрузкой для ДВС является автотранспортное средство (АТС) [1], тогда вращающий момент двигателя уравновешивается моментом сопротивления нагрузки АТС, Нм

$$
M_{c}=M
$$

где $M_{c}=f_{1}\left(V_{a}, \omega\right)$ - момент сопротивления нагрузки АТС (Нм), зависящий от частоты вращения коленчатого вала $\omega\left(\right.$ мин $\left.^{-1}\right)$ и скорости движения автомобиля $V_{a}$ (км/ч). Зависимость $M_{c}$ от указанных параметров может быть получена на основании известных характеристик конкретного двигателя, либо определен расчетным путем

$$
M_{c}=\eta_{g} \cdot P_{t} \cdot \frac{r_{k}}{u \cdot \eta_{t p}}=\eta_{g} \cdot P_{t} \cdot \frac{r_{k} \cdot \omega_{k}}{\omega \cdot \eta_{t p}}
$$

где $P_{t}$ - тяговое усилие автотранспортного средства, оборудованного газовым ДВС (Н); $\eta_{g}$ и $\eta_{t p}-$ коэффициенты деформации колеса и трения качения;

$r_{k}$ - статический радиус колеса (м);

$u$ - передаточное отношение главной передачи и коробки передач

$$
u=\frac{\omega}{\omega_{k}}
$$

$\omega_{\kappa}, \omega-$ угловые скорости колеса и коленчатого вала ДВС, рад/с.

Приращение крутящего момента $\Delta M_{c}$, могут быть вызваны либо приращениями скорости автомобиля $\Delta V_{a}$, либо приращением угловой скорости КВ ДВС $\Delta \omega$

$$
\Delta M_{c}=\frac{\partial M_{c}\left(V_{a 0}, \omega_{0}\right)}{\partial V_{a}} \cdot \Delta V_{a}+\frac{\partial M_{c}\left(V_{a 0}, \omega_{0}\right)}{\partial \omega} \cdot \Delta \omega
$$

Частные производные, определенные в точке, соответствующей выбранному равновесному режиму:

$$
\begin{gathered}
\frac{\partial M_{c}\left(V_{a 0}, \omega_{0}\right)}{\partial V_{a}}=\eta_{g} \cdot P_{t 0} \cdot \frac{r_{k} \cdot \omega_{\kappa}}{\omega_{0} \cdot \eta_{t p} \cdot V_{a 0}} \\
\frac{\partial M_{c}\left(V_{a 0}, \omega_{0}\right)}{\partial \omega}=\eta_{g} \cdot P_{t 0} \cdot \frac{r_{k} \cdot \omega_{\kappa}}{\omega_{0}^{2} \cdot \eta_{t p}}
\end{gathered}
$$


Дифференциальное уравнение приращения скорости вращения коленчатого вала ДВС

\section{для привода автотранспортного средства}

Уравнение, описывающее динамические свойства ДВС, представлено следующей зависимостью

$$
J \frac{d \Delta \omega}{d t}=\Delta M-\Delta M_{c}
$$

где $J$ - приведенный момент инерции вращающихся масс ДВС и автотранспортного средства.

Подставив в данное уравнение выражения для приращений крутящего момента двигателя $\Delta M$ и момента сопротивления $\Delta M_{c}$, создаваемого ведущими колесами автотранспортного средства на силовой агрегат, тогда получим

$$
\begin{aligned}
& J \frac{d \Delta \omega}{d t}+\left(\frac{\partial M_{c}\left(V_{a 0}, \omega_{0}\right)}{\partial \omega}-K \cdot q_{u 0} \cdot \eta_{i 0} \cdot \frac{\partial \eta_{m}\left(\beta_{0}, \omega_{0}\right)}{\partial \omega}\right) \cdot \Delta \omega= \\
& +K \cdot \eta_{m 0} \cdot\left(\eta_{i 0}-\frac{8,314 \cdot p_{i} \cdot V_{h} \cdot \mu}{H_{u} \cdot q_{u 0} \cdot R}\right) \cdot \Delta q_{u}-\frac{\partial M_{c}\left(V_{a 0}, \omega_{0}\right)}{\partial V_{a}} \cdot \Delta V_{a}+K \cdot q_{u 0} \cdot \eta_{i 0} \cdot \frac{\partial \eta_{m}\left(\beta_{0}, \omega_{0}\right)}{\partial \beta} \cdot \Delta \beta+ \\
& +K \cdot q_{u 0} \cdot \eta_{m 0} \cdot \frac{8,314 \cdot p_{i}}{H_{u} \cdot \eta_{v 0} \cdot p_{a 0}} \cdot\left(L_{0} \cdot \alpha_{0} \cdot T_{a} \cdot\left(\frac{1}{p_{a 0}}+\frac{K_{h 2}}{\eta_{v 0}}\right)-\frac{V_{h} \cdot \mu \cdot\left(\eta_{v 0}+K_{h 2} \cdot p_{a 0}\right)}{q_{u 0} \cdot R}\right) \cdot \Delta\left(\Delta p_{a}\right)
\end{aligned}
$$

Величина

$$
F_{\text {д }}=\frac{\partial M_{c}\left(V_{a 0}, \omega_{0}\right)}{\partial \omega}-K \cdot q_{u 0} \cdot \eta_{i 0} \cdot \frac{\eta_{m}\left(\beta_{0}, \omega_{0}\right)}{\partial \omega}
$$

- представляет собой фактор устойчивости режима работы двигателя.

Для удобства анализа и сопоставления переходных процессов, а также оценки динамических показателей двигателя, запишем его уравнение в безразмерной форме.

После приведения уравнения к стандартной форме записи, получим

$$
T_{\text {д }} \cdot \frac{d \bar{\omega}}{d t}+\bar{\omega}=k_{g} \cdot \overline{q_{u}}+k_{\beta} \cdot \bar{\beta}-k_{v} \cdot \overline{V_{a}}+k_{p} \cdot \overline{p_{a}}
$$

Относительные координаты уравнения (47) представлены в виде

$$
\bar{\omega}=\frac{\Delta \omega}{\omega_{0}} ; \bar{g}_{u}=\frac{\Delta g_{u}}{g_{u 0}} ; \overline{V_{a}}=\frac{\Delta V_{a}}{V_{a 0}} ; \bar{p}_{a}=\frac{\Delta\left(\Delta p_{a}\right)}{\Delta p_{a 0}}
$$

где $\bar{\omega}$ - безразмерное изменение угловой скорости вращения вала ДВС;

$\overline{q_{u}}-$ безразмерное изменение цикловой подачи топлива;

$\overline{V_{a}}$ - безразмерное изменение скорости движения АТС;

$\overline{p_{a}}$ - безразмерное изменение разряжения рабочего тела во впускном коллекторе;

$\Delta$ - элементарное изменение параметров при переходе от одного установившегося режима на другой; параметры с индексом 0 , снятые на установившихся режима эксплуатации АТC.

Здесь $T_{\text {д }}=\frac{J \cdot \omega_{0}}{\left(\frac{\partial M_{c}\left(V_{a 0}, \omega_{0}\right)}{\partial \omega}-K \cdot q_{u 0} \cdot \eta_{i 0} \cdot \frac{\partial \eta_{m}\left(\beta_{0}, \omega_{0}\right)}{\partial \omega}\right)}-$ постоянная времени ДВС,

характеризует скорость реакции газового двигателя в процессе движения АТC; 


$$
K_{\beta}=\frac{\left(K \cdot q_{u 0} \cdot \eta_{i 0} \cdot \beta_{0} \cdot \frac{\partial \eta_{m}\left(\beta_{0}, \omega_{0}\right)}{\partial \beta}\right) \cdot \omega_{0}}{\left(\frac{\partial M_{c}\left(V_{a 0}, \omega_{0}\right)}{\partial \omega}-K \cdot q_{u 0} \cdot \eta_{i 0} \cdot \frac{\partial \eta_{m}\left(\beta_{0}, \omega_{0}\right)}{\partial \omega}\right)}-\text { безразмерный коэффициент, }
$$

характеризующий влияние изменения углового положения дроссельной заслонки на приращение скорости вращения КВ ДВС;

$$
K_{g}=\frac{K \cdot \eta_{m 0} \cdot q_{u 0} \cdot\left(\eta_{i 0}-\frac{8,314 \cdot p_{i} \cdot V_{h} \cdot \mu}{H_{u} \cdot q_{u 0} \cdot R}\right) \cdot \omega_{0}}{\left(\frac{\partial M_{c}\left(V_{a 0}, \omega_{0}\right)}{\partial \omega}-K \cdot q_{u 0} \cdot \eta_{i 0} \cdot \frac{\partial \eta_{m}\left(\beta_{0}, \omega_{0}\right)}{\partial \omega}\right)}-\text { безразмерный коэффициент, }
$$

характеризующий влияние приращения цикловой подачи топлива на приращение скорости вращения КВ ДВС;

$$
K_{V}=\frac{\frac{\partial M_{c}\left(V_{a 0}, \omega_{0}\right)}{\partial V_{a}} \cdot \omega_{0}}{\left(\frac{\partial M_{c}\left(V_{a 0}, \omega_{0}\right)}{\partial \omega}-K \cdot q_{u 0} \cdot \eta_{i 0} \cdot \frac{\partial \eta_{m}\left(\beta_{0}, \omega_{0}\right)}{\partial \omega}\right)}-\text { безразмерный коэффициент, }
$$

характеризующий влияние изменения скорости движения АТС на приращение скорости вращения КВ ДВС;

$$
K_{p}=\frac{K \cdot q_{u 0} \cdot p_{a 0} \cdot \eta_{m 0} \cdot \frac{8,314 \cdot p_{i}}{H_{u} \cdot \eta_{v 0} \cdot p_{a 0}} \cdot\left(L_{0} \cdot \alpha_{0} \cdot T_{a} \cdot\left(\frac{1}{p_{a 0}}+\frac{K_{h 2}}{\eta_{v 0}}\right)-\frac{V_{h} \cdot \mu \cdot\left(\eta_{v 0}+K_{h 2} \cdot p_{a 0}\right)}{q_{u 0} \cdot R}\right) \cdot \omega_{0}}{\left(\frac{\partial M_{c}\left(V_{a 0}, \omega_{0}\right)}{\partial \omega}-K \cdot q_{u 0} \cdot \eta_{i 0} \cdot \frac{\partial \eta_{m}\left(\beta_{0}, \omega_{0}\right)}{\partial \omega}\right)}-
$$

безразмерный коэффициент, характеризующий влияние приращения разрежения во впускном коллекторе на приращение скорости вращения КВ ДВС.

В операторной форме записи уравнение (47) будет иметь вид

$$
\left(T_{\text {д }} \cdot p+1\right) \cdot \bar{\omega}=k_{g} \cdot \overline{q_{u}}+k_{\beta} \cdot \bar{\beta}-k_{v} \cdot \overline{V_{a}}+k_{p} \cdot \overline{p_{a}}
$$

Где, двучлен в скобках, взятый из уравнения (49)

$$
d_{\text {д }}(p)=\left(T_{\text {д }} \cdot p+1\right)
$$

- является собственным оператором газового двигателя.

Упрощенное изображение структурной схемы газового двигателя приведено на рис. 1.

C помощью вышеприведенного операторного уравнения первой степени, можно исследовать динамические свойства переходных процессов газовых ДВС, которые используются в качестве силовых агрегатов для привода автотранспортных средств (ATC).

Рациональный подбор типа газо-топливной аппаратуры и узлов воздухоснабжения улучшает динамические характеристики газовых двигателей нового поколения.

От эффективной и согласованной работы систем воздухоснабжения, зажигания и топливоподачи, установленных в силовых агрегатах, снижается инерционность переходных процессов протекающих в цилиндрах ДВС. 


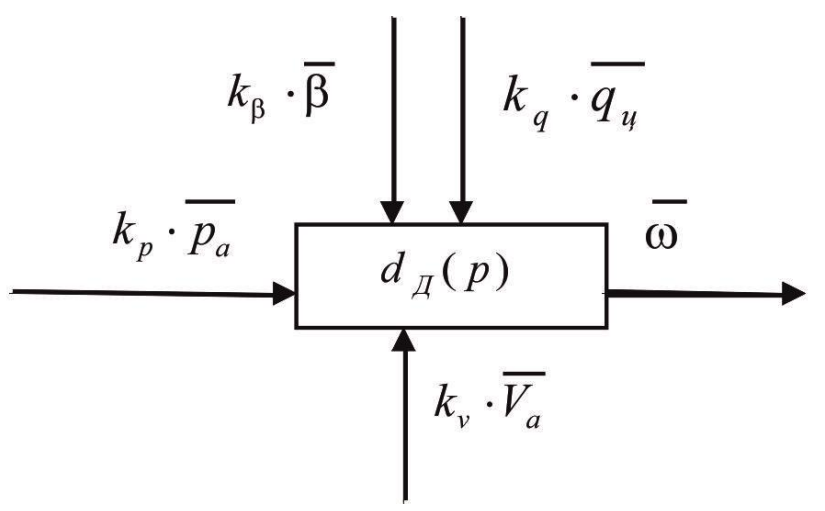

Рис. 1. Упрощенное изображение структурной схемы газового двигателя

Своевременная подача воздуха и топлива в цилиндры ДВС согласованная с моментом подачи искры системой зажигания существенно улучшают характеристики процесса сгорания (динамику и характер выгорания топлива, моменты достижения максимальных давлений и температур цикла), способствуют улучшению протекания рабочего процесса в цилиндрах силового агрегата в целом.

\section{Выводы.}

1. Полученное ДУ, описывает переходные процессы изменения угловой скорости коленчатого вала газового ДВС транспортного средства в широком диапазоне скоростных и нагрузочных режимах его работы.

2. Получено линейное неоднородное ДУ 1-го порядка с постоянными коэффициентами, которое можно использовать для разработки и реализации математических моделей многоконтурных функциональных систем транспортного ДВС.

\section{ЛИТЕРАТУРА}

1. Крутов В. И. Двигатель внутреннего сгорания как регулируемый объект / В. И. Крутов. - М.: Машиностроение. - 1978. - 472 с.

2. Иванов-Смоленский А. В. Электрические машины: Учебник для вузов / А. В. Иванов-Смоленский. М.: Энергия. $-1980 .-928$ с.

3. Система всережимного регулирования наддува автомобильного дизеля / В. М. Манойло // Двигатели внутреннего сгорания. -1997. - Вып. 56-57, С. 197-203.

4. САР турбонаддува ДВС с применением перепуска газа / Г. В. Сиволап, Ю. Г. Рассудов, А. Г. Рыбальченко // Двигатели внутреннего сгорания. -1975. - Вып. 22, С.47-52.

5. Математическое моделирование переходных процессов в регулируемой системе “ДВС - волновой обменник давления" / А. С. Куценко, В. М. Манойло // Автомобильный транспорт: Сборн. научн. трудов, - Вып.6, - Харьков: ХГАДТУ, 2000. - С.59-61.

6. Анализ динамики САР турбонаддува ДВС/ Г. В. Сиволап, Ю. Г. Рассудов, А. Г. Рыбальченко // Двигатели внутреннего сгорания. - 1975. - Вып. 22, С. 52-55.

7. Крутов В. И. Регулирование турбонаддува ДВС: Учебное пособие для вузов / Крутов В. И., Рыбальченко А. Г. - М.: Высшая школа. - 1978. - 213 с. 